\title{
Multi-Joint Reaching Movements and Eye-Hand Tracking in Cerebellar Incoordination: Investigation of a Patient with Complete Loss of Purkinje Cells
}

\author{
W.J. Becker, B.L. Morrice, A.W. Clark and R.G. Lee
}

\begin{abstract}
Performance on an eye-hand tracking task and a multi-joint reaching movement to a visual target was studied in a patient with stable cerebellar ataxia and in control subjects. The patient subsequently died and a full neuropathological examination was performed. The neuropathological findings were similar to those seen in patients with paraneoplastic cerebellar degeneration, but no tumor was found at autopsy eight years after onset of the patient's cerebellar syndrome. A severe cerebellar cortical degeneration with complete Purkinje cell loss was demonstrated, whereas cerebellar nuclei and brainstem structures showed no neuronal loss. Tracking performance by the patient was characterized by abnormally large numbers of high velocity movements and hand direction reversals, and by excessive lagging of the hand behind the target in time. In the multi-joint reaching movement, the patient showed a delay in movement onset at the elbow joint compared to movement onset at the shoulder joint. The velocity profile of the movement at the shoulder joint was abnormal. The duration of the acceleration phase was poorly correlated with both peak angular velocity and the duration of the deceleration phase. One of the most striking findings was the inability of the patient to consistently produce the same movement direction from trial to trial while reaching to the same target. Our data suggests that the cerebellar cortex is involved in multiple aspects of motor control including visuomotor integration mechanisms.
\end{abstract}

\begin{abstract}
RÉSUMÉ: Mouvements polyarticulaires ciblés et poursuite oeil-main dans l'incoordination cérébelleuse: investigation d'un patient avec perte complète des cellules de Purkinje Nous avons étudié la performance d'un patient avec une ataxie cérébelleuse stable et de sujets contrôles lors de 1'accomplissement d'une épreuve de poursuite oeilmain et d'un mouvement polyarticulaire pour atteindre une cible visuelle. Le patient est décédé ultérieurement et un examen neuropathologique complet a alors été effectué. Les constatations neuropathologiques ont été semblables à celles qu'on retrouve chez les patients avec une dégénérescence cérébelleuse paranéoplasique, mais aucune tumeur n'a été retrouvée à l'autopsie huit ans après le début du syndrome cérébelleux. Une dégénérescence corticale cérébelleuse sévère avec perte complète des cellules de Purkinje a été mise en évidence, alors que les noyaux cérébelleux et les structures du tronc cérébral ne présentaient aucune perte neuronale. L'épreuve de poursuite effectuée par le patient était caractérisée par une quantité anormale de mouvements de haute vélocité et de changements de direction de la main, et par un retard excessif de la main par rapport à la cible. Dans le mouvement polyarticulaire pour atteindre la cible, le patient manifestait un retard à débuter le mouvement au niveau du coude comparé au début du mouvement à l'épaule. Le profil de vélocité du mouvement à l'épaule était anormal. La durée de la phase d'accélération était peu corrélée à la vélocité angulaire de pointe et à la durée de la phase de décélération. Une des constatations les plus frappantes était l'inhabilité du patient à reproduire avec constance la même direction du mouvement d'un essai à l'autre lorsqu'il tentait d'atteindre la même cible. Nos données suggèrent que le cortex cérébelleux est impliqué dans plusieurs aspects du contrôle moteur, incluant les mécanismes d'intégration visuomoteurs.
\end{abstract}

Can. J. Neurol.Sci. 1991; 18: 476-487

The cerebellum plays an important role in coordination of movement, but its exact contribution is still unclear. Over half a century ago, Gordon Holmes concluded that the cerebellum was involved in both regulation of postural tone and in reinforcement or tuning of the motor cortex during fast volitional move- ments. ${ }^{1}$ As Holmes demonstrated, careful analysis of motor deficits in patients with lesions of the cerebellum can increase our understanding of the functional role of the cerebellum during normal movements. Even with modern imaging techniques however, it is not always evident exactly which parts of the

From the Department of Clinical Neurosciences, The University of Calgary, Calgary

Received February 5, 1991. Accepted in final form May 23, 1991

Reprint requests to: Dr. W.J. Becker, Calgary General Hospital, Room M4-022, 841 Centre Ave. E., Calgary, Alberta, Canada T2E 0Al 
cerebellum have been damaged in these patients, and whether the damage involves the deep cerebellar nuclei as well as the cerebellar cortex.

We report here the results of motor control studies carried out on a patient with cerebellar dysfunction who subsequently died of an acute unrelated illness, and had full neuropathological examination of the central nervous system. Microscopic examination showed virtual absence of Purkinje cells and some associated loss of the granule cell layer, but the deep cerebellar nuclei were intact, as was the inferior olive.

We have evaluated our studies to assess what abnormalities in movement control resulted from our patient's very selective degeneration of the cerebellar cortex. The results presented here derive from a visuomotor tracking task and a multi-joint reaching movement to a visual target in three dimensional space.

\section{Case History}

The patient was first seen by one of us (RGL) in July 1980 at age 27 . Over several days she had developed headache, dysarthria, incoordination of the arms and legs, and severe gait ataxia. Neurologic examination at that time showed a severe disturbance of cerebellar function with relative sparing of other components of the central nervous system. A brain CT scan was normal. CSF showed 128 lymphocytes per cubic $\mathrm{mm}$, a protein of $0.40 \mathrm{gms} / \mathrm{L}$, and a borderline $\mathrm{IgG}$ at $15.4 \%$ of total CSF protein. CSF oligoclonal bands were present. As the patient had never had previous neurologic problems, the clinical diagnosis at that time was an acute viral encephalitis localized to the cerebellum, or possibly a first attack of multiple sclerosis.

Over the next several weeks she improved slowly, but was eventually left with severe cerebellar deficits which persisted without change for the next seven years until her death. Our physiological studies were done over five years after onset of her symptoms, at which time the neurologic deficits were stable. Clinical examination showed slow dysarthric speech, mild gaze-evoked nystagmus and irregular pursuit eye movements. Strength was normal in all muscle groups, but muscle tone was reduced in the arms and legs. Both upper limbs showed severe incoordination and dysmetria and slowing of rapid alternating movements and fine finger movements without definite intention tremor. The lower extremities showed marked incoordination on performing the heel-shin test. She was unable to stand or walk without support. When she attempted to walk with assistance, she was markedly ataxic and showed irregular poorly controlled movements of both legs. Tendon reflexes were moderately brisk and bilaterally symmetrical; plantar responses were both downgoing. Sensory examination was normal.

The patient died suddenly in May 1988 of aspiration and asphyxiation following a presumed epileptic seizure.

\section{Pathological Findings}

The brain was immersion-fixed in buffered formalin for two weeks. Subsequently, blocks of tissue were imbedded in paraffin and 6-micron thick sections were stained with hematoxylineosin, luxol fast blue (LFB), Bielschowsky silver, and cresyl violet stains. Two postmortem brains, from individuals aged 37 and 38 years, were similarly processed as controls.

The general autopsy revealed no evidence of carcinoma. The brain weighed 1347 grams. Gross examination revealed no convincing abnormalities except a thin recent subdural hematoma. The cerebellum did not appear grossly atrophic. On microscopic examination, however, virtually total loss of Purkinje cells was apparent (Figure 1, A and B). Granule cells were also depleted, but much less affected than Purkinje cells. Survival of basket cells was documented by the numerous empty baskets on Bielschowsky-stained sections. Golgi II cells were also readily identifiable. The cerebellar white matter showed mild degenerative changes consistent with loss of cortical neurons. No loss of neurons was apparent in the dentate nucleus (Figure 1C). The reduced width of the dentate probably reflected a dramatic loss of afferent fibre terminals. These changes were not associated with inflammation or demyelinating lesions. Outside the cerebellum, histologic changes were very subtle or absent. The superior cerebellar peduncle was normal, with no apparent loss of myelinated fibres in LFB-stained sections. The nuclei basis pontis and inferior olive revealed no apparent loss of neurons. There was a mild gliosis and slight reduction in overall size of the olive when compared with age-matched controls, probably related to retrograde changes in this structure following damage to its field of projection.

In summary, the pathological examination showed selective degeneration of the cerebellar cortex with apparent complete loss of Purkinje cells. In contrast, the deep cerebellar nuclei showed only minor changes, with no evidence of neuronal loss.

\section{MethodS}

\section{Reaching Movements}

Three normal subjects (two males and one female) aged 2730 were studied in addition to the patient. The protocols were approved by the Conjoint Medical Ethics Committee of The University of Calgary and informed consent was obtained in all cases.

The subject sat in a chair with the right arm supported on an arm-rest, so that the upper arm was vertical and the elbow flexed to ninety degrees. In response to an auditory tone, the subject reached out and touched with the index finger a small target placed at shoulder height directly in front of the subject. The target was $0.5 \mathrm{~cm}$ in diameter, and was placed approximately $7 \mathrm{~cm}$ closer than the maximum distance the subject could reach without leaning forward. The subjects were asked to move as quickly as possible for some trials, and at a "natural speed" or even more slowly for other trials so that a variety of movement velocities could be studied.

Infra-red light-emitting diodes (IREDs) were placed on the subject's arm at the shoulder, elbow, wrist and index fingertip, and on the target. During the reaching movement, the position of the IREDs was monitored by a two camera Watsmart motion analysis system. For studies of joint angles, the elbow angle was determined from the relative positions of the three IREDs placed over the shoulder, elbow, and wrist. The shoulder angle was defined as the angle between a straight line joining the shoulder and elbow IREDs and the vertical plane running through the shoulder IREDs.

This study focused on coordination between the elbow and the shoulder joint and the data with regard to the wrist joint and fingers will not be considered in detail. Analysis of movement direction focused on the wrist IRED rather than the finger IRED in order to assess the ability of the central nervous system to control a two joint system.

IRED positions over time, joint angular velocities and tangential velocities for individual IREDs were displayed on a graphics terminal and measurements made by means of cursors. To determine the movement direction specified by the first part of the motor program, we measured the movement direction between the attainment of $50 \%$ peak velocity and peak velocity, 
PATIENT

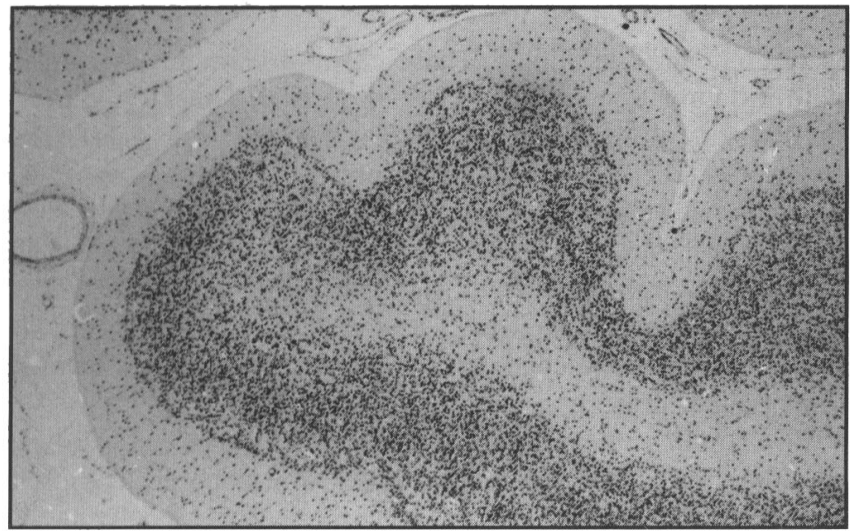

A
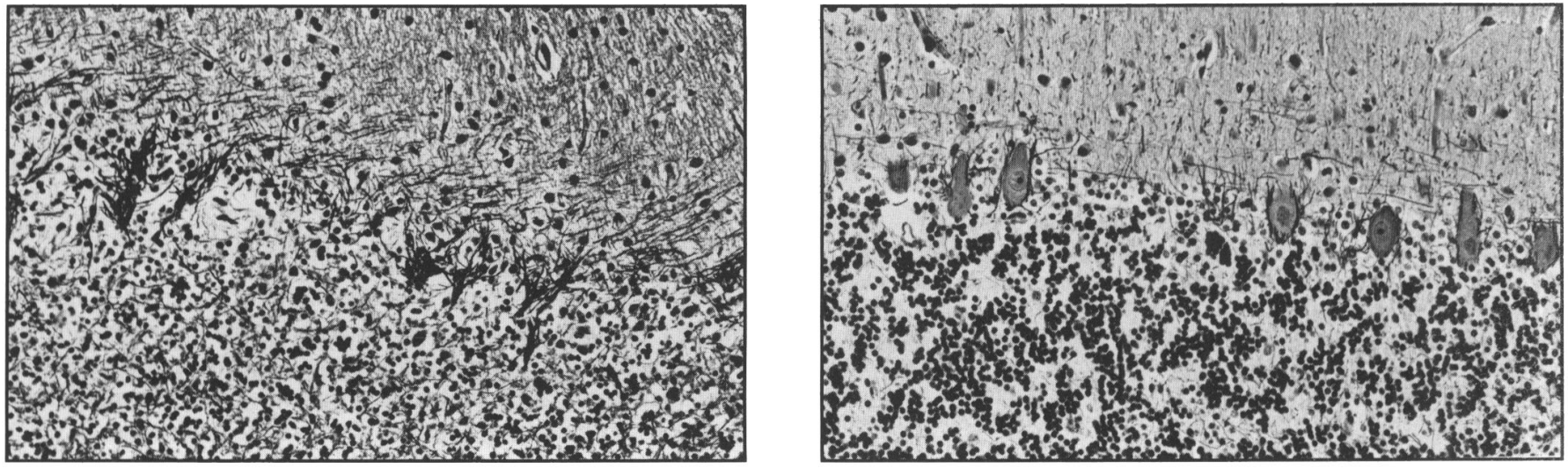

B
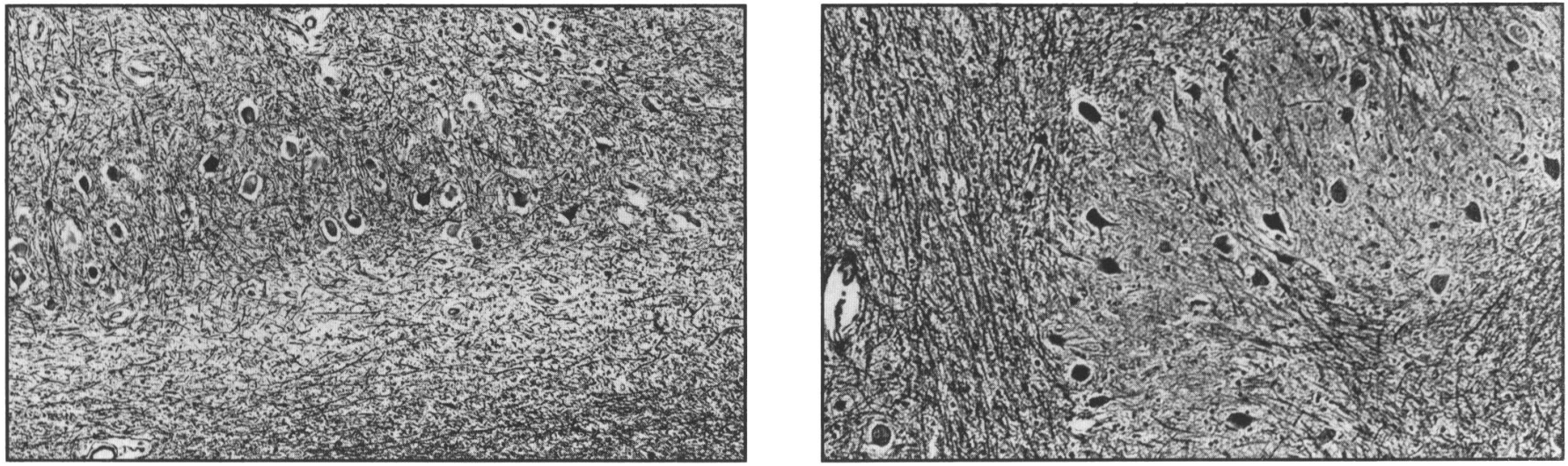

C

Figure $1-(A)$ In the cerebellar folia shown, the patient shows narrowing of the molecular layer and attenuation of the granule cells. No normal Purkinje cells were identified in the length of the Purkinje cell layer shown here for the patient, whereas the normal control specimen shows approximately 20 Purkinje cells. Nissl stain, final magnification 50X. (B) The cerebellar cortex of the patient showed empty baskets at the sites of Purkinje cell loss. The control specimen shows normal relationship of Purkinje cells to basket fibres. Bielschowsky silver stain, 230X. (C) The dentate nucleus of the patient shows a population of dentate neurons with no obvious cell loss. The width of the dentate nucleus appears decreased and the density of the argyrophilic fibres increased as compared to the control specimen. Bielschowsky silver stain, $115 X$. 
using the tangential velocity of the wrist IRED. This excluded the variable early components of the movement and later portions which often included corrective movements. The movement direction was expressed as the slopes of the wrist IRED path in the horizontal and vertical planes. For measurements of peak velocity magnitude and timing, the shoulder and elbow angular velocities were displayed over time on the graphics terminal and measurements made with a cursor for each movement trial. Unless otherwise stated, velocity measurements refer to joint angular velocity. For analysis of movement timing, including timing of peak velocity, movement onset, and velocity profile analysis, only faster movements with a peak angular shoulder velocity of more than $150 \mathrm{deg} . / \mathrm{sec}$. were studied. The onset of movement at each joint was defined as the time at which the angular velocity reached $5 \%$ of the peak velocity for that movement trial.

The velocity profiles were analyzed by determining the magnitude and timing of peak angular velocity at the shoulder joint and then measuring the time intervals between peak velocity and the points at which $50 \%$ peak velocity was reached during the acceleration and deceleration phases.

\section{Eye-Hand Tracking}

In addition to the patient, five healthy control subjects (age 16-29) were studied in these experiments. The methods used for the tracking task have been described in detail ${ }^{2}$ but will be summarized here. Subjects were seated with the forearm supported in a manipulandum that restricted movement to the wrist joint. A vertical rod coupled the manipulandum to a torque motor. Wrist angle was monitored by a precision potentiometer and the signal from the potentiometer was differentiated to obtain wrist angular velocity. The subjects were presented with a visual display on a CRT screen which included a target that moved horizontally on the screen in an unpredictable manner for twenty seconds, and a cursor that the subject controlled by flexing and extending the wrist joint. Target velocities ranged from $0-30$ degrees per second.
A viscous load was provided during eight of the sixteen tracking trials performed by the subject by feeding back the velocity of the handle movement to the amplifier that controlled the torque motor. The remaining eight tracking trials were performed with no added load. To prevent possible learning effects from affecting the comparison between loaded and unloaded tracking trials, groups of loaded trials were intermingled with groups of unloaded trials in order of performance. Three different levels of viscous loading were used $(0.044,0.031$, and 0.022 Newtons/sec/deg).

At each data point, the absolute value of the difference between the target and cursor position was measured. These values were then summed for each twenty second trial to create a quantitative measure of error or accuracy, which was referred to as the positional error score.

The velocity records were analyzed by measuring the number of velocity peaks exceeding 30 degrees/sec and 50 degrees/sec. The number of baseline crossings (reversals) in the velocity trace were also counted.

The average time lag between target and hand movement for individual twenty second tracking trials was computed by performing sequential time shifts of all the data points representing cursor position with respect to target position. The positional error score was recalculated following each time shift of the data points until a point was identified at which the positional error score reached a minimum value.

Student's t-test was used for all statistical comparisons.

\section{RESULTS}

\section{Reaching Movements \\ Movement Direction}

Figure 2 shows examples of movement paths for a normal subject and for the patient while they were reaching out to touch a target with the index finger. Three movement trials are superimposed. The paths of the IRED's attached to the wrist and
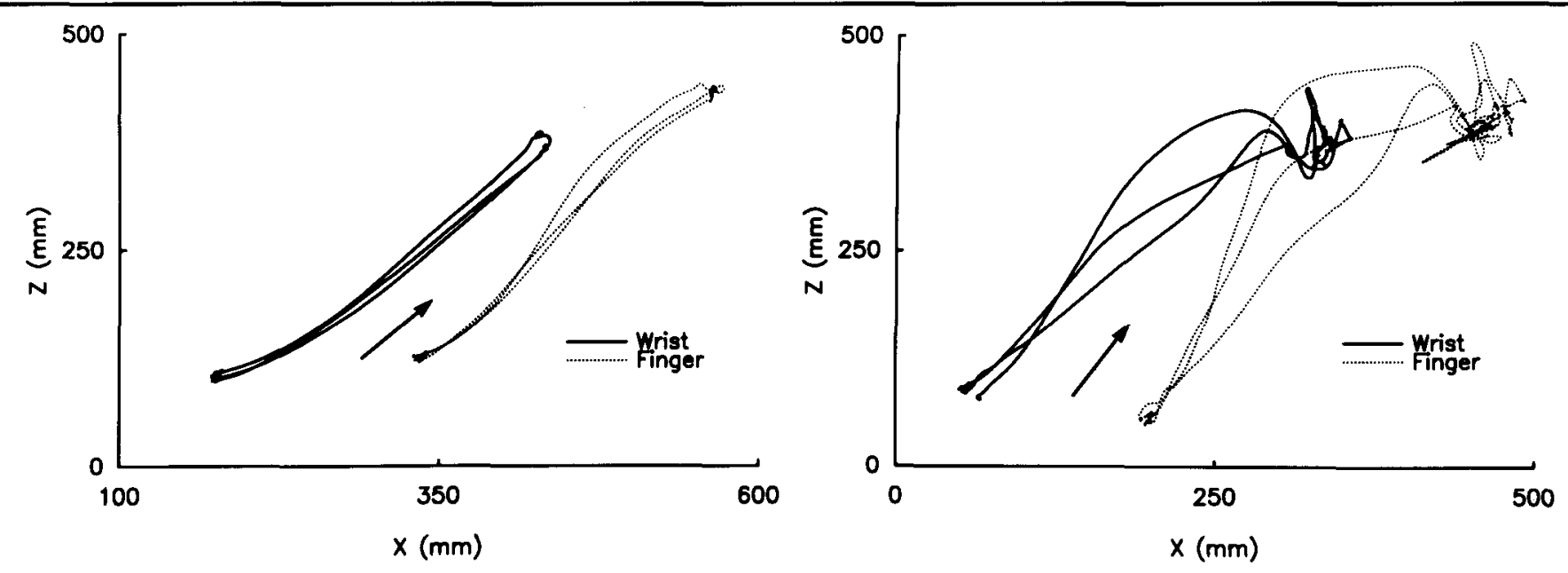

Figure 2 - The left panel shows movement paths in the X-Z plane for three movement trials in a control subject for the wrist IRED (solid lines) and the index finger tip IRED (dotted lines). The right panel shows in a similar manner three movement trials by the patient. $X$ indicates the forward direction relative to the subject. $\mathrm{Z}$ indicates the vertical direction. All movements begin in the lower left portion of the panel, and proceed in the direction indicated by the arrows. For the patient, movement direction both at the wrist and index finger was much more variable from trial to trial. 
index finger are shown in the $x-z$ plane (viewing the subject from the side).

In normal subjects performing a reaching task of this type, movements at the shoulder and elbow joints were coupled such that the finger and wrist moved in either a straight line or a slightly curved path. The initial direction of movement was very consistent from trial to trial; there was slight variation in the later part of the movement path.

In the patient a number of abnormalities were apparent. There was marked variability in movement direction from trial to trial and the paths for the finger and wrist were not straight. In the examples shown in Figure 2 there was a tendency for the initial component of the movement to be in a more vertical or upward direction than in normal subjects. This was followed by a horizontal component and, in fact, some movements appeared to have two distinct components. In addition, as the finger approached the target there were numerous coarse, irregular terminal oscillations.

The marked variability in initial movement direction is illustrated in Figure 3 which shows the standard deviations for the slope of the wrist movement paths for the patient and for the three control subjects. In both the vertical and horizontal planes the standard deviations for the slope of the movement paths were almost 10 times greater in the patient than in the control subjects. For both the patient and the controls there was less variability in movement direction in the horizontal than in the vertical plane.

\section{Coupling of Movement at the Shoulder and Elbow}

Figure 4 illustrates the relationships between movement at the shoulder and elbow joints. In control subjects the elbow began to extend at almost the same time as the onset of shoulder flexion, and angular changes at these two joints occurred simultaneously. Plotting elbow angle against shoulder angle produced a slightly curved line which was consistent from trial to trial.

In the patient there was often a considerable delay between the onset of movement at the shoulder and the beginning of elbow extension. The mean time interval between onset of movement at the shoulder and elbow was $264 \pm 23 \mathrm{msec}$. (N=11 trials) This was significantly different $(p<0.001)$ from the comparable mean value for control subjects ( $23 \pm 29 \mathrm{msec} ; \mathrm{N}=17$ ).

The elbow angle - shoulder angle plots for the patient (Figure 4b) showed an irregular relationship with much variability from trial to trial. The initial segment of these curves was relatively flat, reflecting the tendency for considerable shoulder

Figure $3-(A)$ The method used to calculate the slope of a movement direction is shown for the vertical plane. The solid line represents the path of the wrist IRED during the initial portion of a movement trial. $Z$ indicates the upward direction, $X$ indicates the forward direction relative to the subject. For movement direction in the horizontal $(X-Y)$ plane, $\Delta y / \Delta x$ was used in a similar fashion to calculate the "horizontal" slope. $(B)$ The standard deviation for the slope of the wrist IRED path is shown for the vertical plane (middle panel) and the horizontal plane (lower panel). " $N$ " indicates a normal subject, and " $P$ " indicates the patient. Ten consecutive movement trials were analyzed for each normal subject, and twenty consecutive movement trials for the patient. The standard deviations of the slopes for the patient movement trials were much larger, indicating much more variability in movement direction from trial to trial while reaching to the same target.

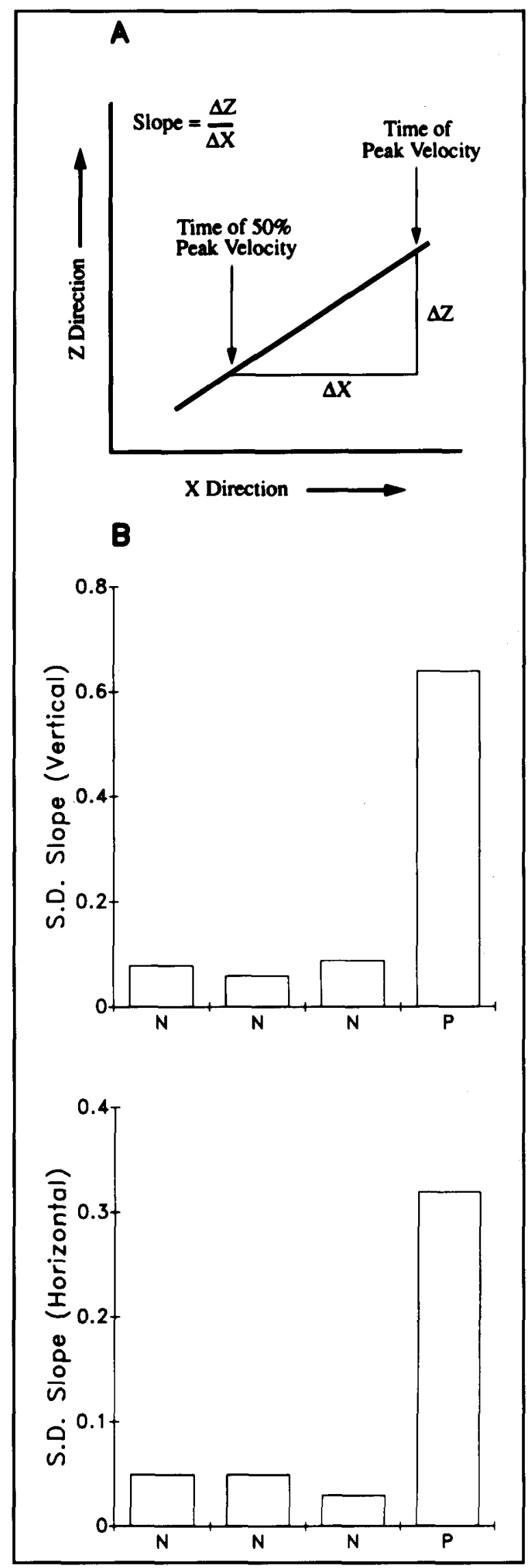


flexion to occur before onset of elbow extension. This altered relationship between movements of the shoulder and elbow was one of the main factors accounting for the abnormal initial trajectory of the finger and hand.

\section{Angular Velocities at Shoulder and Elbow}

Many of the characteristics of the coupling of movement at the shoulder and elbow become apparent on inspection of the timing and magnitude of peak angular velocities at these joints.

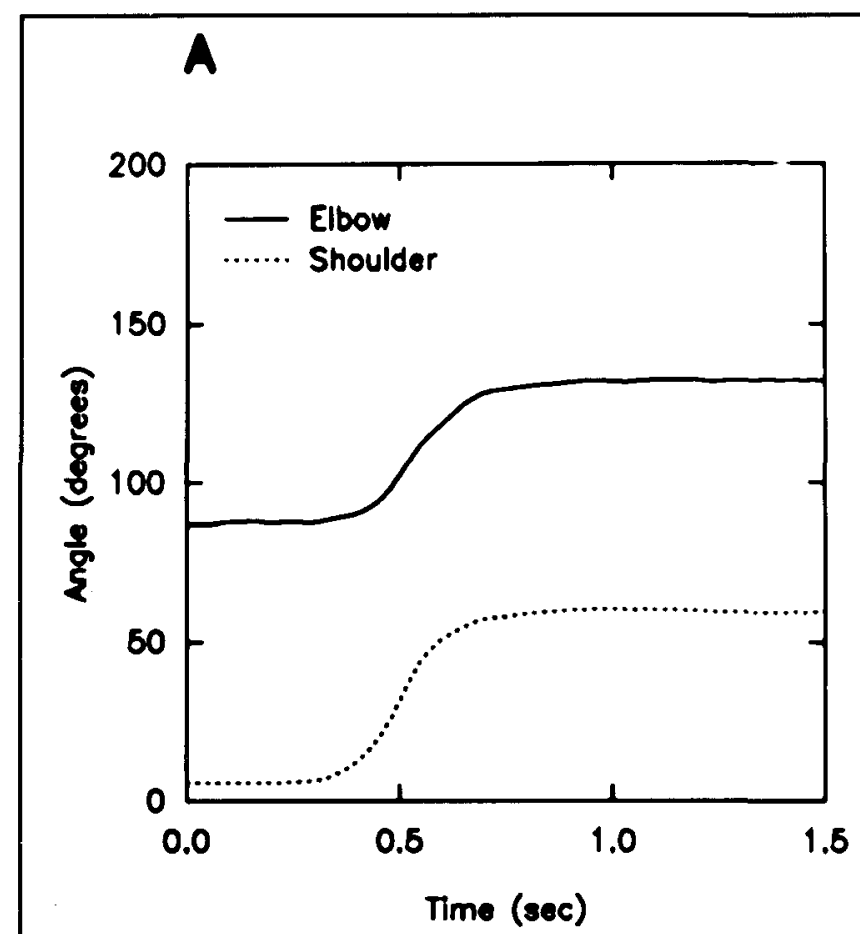

$\mathbf{B}$
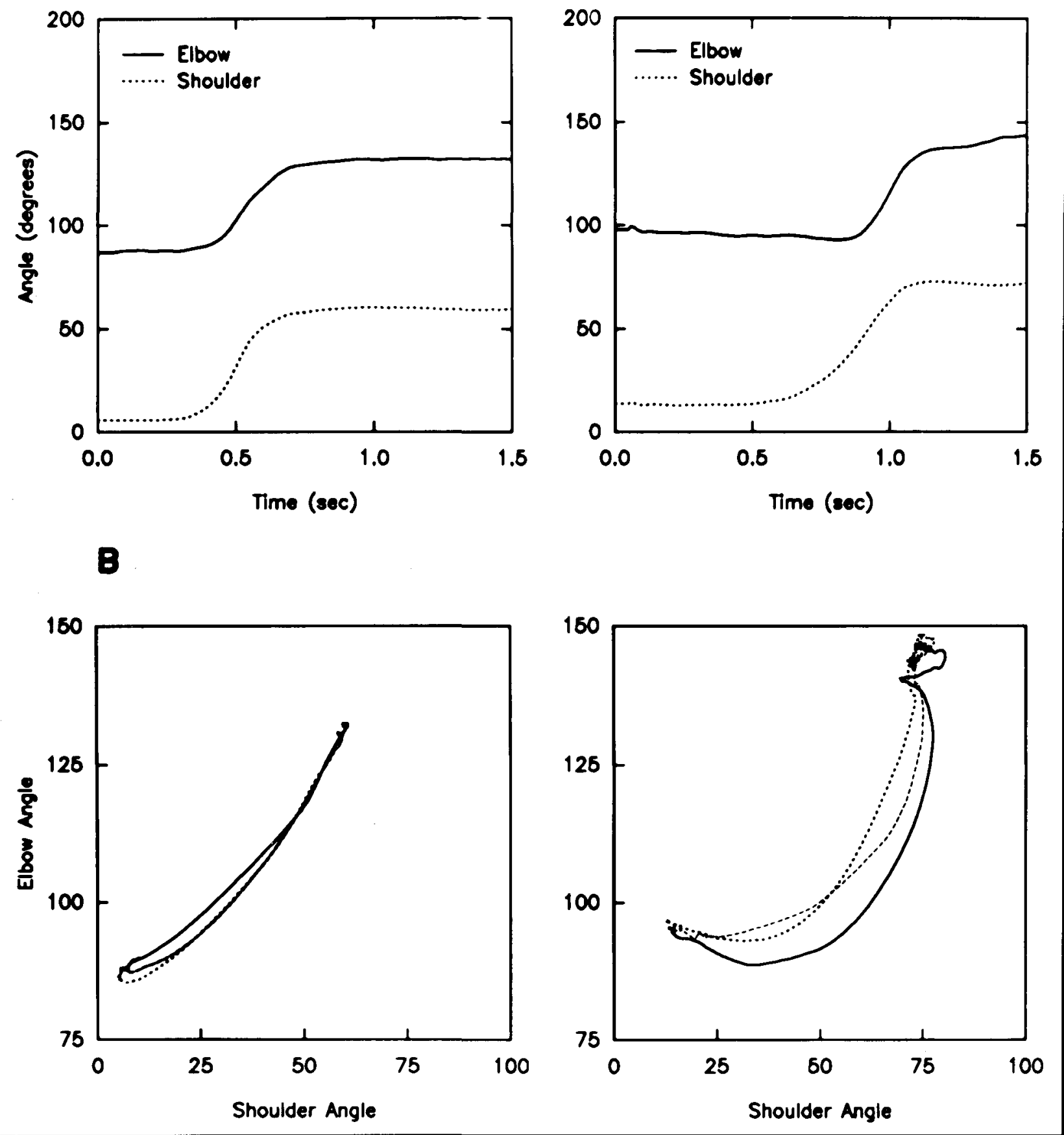

Figure 4-(A) Changes in the elbow and shoulder angle over time are shown for single movement trials for a normal subject (left panel) and for the patient (right panel). In the patient, flexion at the shoulder joint began well before elbow extension, while in the normal subject the two movements began almost simultaneously. (B) Elbow angle is plotted against shoulder angle for three movement trials for a normal subject (left panel) and for the patient (right panel). In the patient, the shoulder angle often changed a considerable amount before elbow extension began. The joint angle relationships were also much more variable from trial to trial for the patient than for the control subject. 
Table 1: Analysis of Angular Velocities at the Shoulder and Elbow During Pointing Movements For 3 Control Subjects and For a Patient With Cerebellar Damage

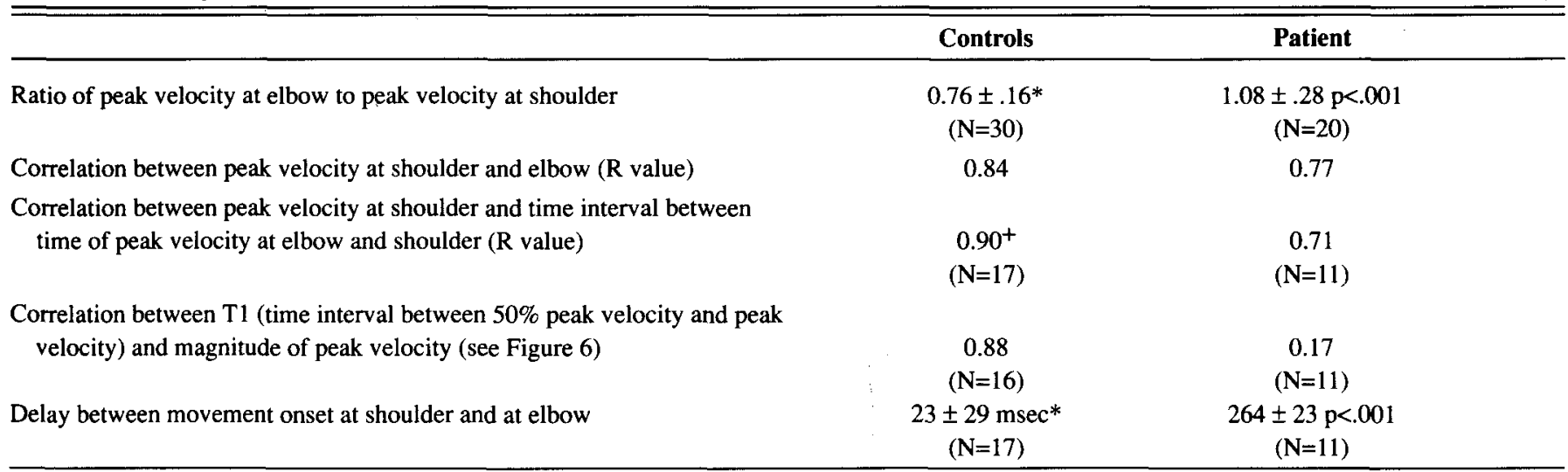

* Values shown are the mean \pm standard deviation. $\mathrm{N}$ refers to the number of movement trials. Values for controls represent the average of all trials measured for 3 subjects.

+ Mean correlations coefficient for the 3 control subjects.

Measurements of several properties of peak angular velocities for the patient and for the control subjects are summarized in Table 1 and in Figures 5 and 6.

In control subjects, for any given movement trial, maximum angular velocity at the elbow was less than at the shoulder. The mean ratio of these angular velocities for the three controls was $0.76 \pm .16$. This relationship was quite variable for the patient, but for 20 trials the mean ratio of elbow velocity to shoulder velocity was $1.08 \pm .28$. The fact that angular velocities at the two joints did not differ in the patient as in normals may be related to the observation that onset of movement at the elbow in the patient occurred relatively later than at the shoulder (Figure 4) and a higher angular velocity would therefore be required for the elbow to "catch up" during the movement.

In both the patient and in the control subjects, for any given movement trial, there was a strong correlation between the magnitude of peak velocity at the shoulder and at the elbow ( $R=0.84$ for controls; 0.77 for patient - see Figure 5). This indicates that movements with faster peak velocities at the shoulder had faster peak velocities at the elbow as well.

In control subjects the time at which peak velocity occurred at the elbow was invariably later than peak velocity at the shoulder. With faster movements this time interval became shorter and there was a strong inverse correlation of the interval between velocity peaks with the magnitude of peak velocity at the shoulder for each of the control subjects $(R=0.81,0.90$, 0.99 ). This coordination of the timing of velocity peaks was preserved in the patient although the relationship was slightly more variable $(\mathrm{R}=0.71)$.

In control subjects faster movements were associated with narrower velocity profiles and a more rapid rise to peak angular velocity. There was a strong inverse correlation between $\mathrm{T} 1$ (time interval between attainment of 50\% peak velocity and peak velocity) and the magnitude of peak angular velocity at the shoulder $(R=0.88)$. For the patient there was no significant correlation between these variables. $(R=0.17$; see Figure $6 c)$.

In the control subjects angular velocity profiles were relatively symmetrical so that there was a constant relationship between $\mathrm{T} 1$ and $\mathrm{T} 2$ (time interval between attainment of peak velocity and $50 \%$ peak velocity on the deceleration phase). Analysis of 12 movement trials from two control subjects with a wide range of velocities gave an $R$ value of 0.84 for each subject. For the patient there was no consistent correlation between $\mathrm{T} 1$ and $\mathrm{T} 2(\mathrm{R}=0.18)$.

\section{Eye-Hand Tracking}

Figure 7 shows results obtained from a control subject and from the patient while they were attempting to track a moving visual target using flexion - extension movements at the wrist. Both the patient and the control subject used a series of multiple small discrete movements to track the target. Inspection of the wrist velocity traces indicates that the frequency of these movements was within the $2-2.5 \mathrm{~Hz}$. range.

The patient tended to lag behind the target more than the control subject and tracking was much less accurate. The mean positional error score obtained by integrating the difference between target and cursor position for the $20 \mathrm{sec}$ tracking trials without a viscous load was $152,275 \pm 17,830$ for the patient, compared to a mean of $31,852 \pm 4,666$ for the five control subjects who participated in this experiment.

Several factors may have contributed to the patient's poor tracking performance. In comparison to normal controls, the patient showed much more frequent reversals in movement direction. The target reversed direction 14 times during each 20 second tracking trial, and theoretically only 14 direction reversals were necessary to accurately tract the target. For control subjects the mean number of direction reversals was 17 , while the patient reversed directions a mean of 41 times $(p<0.005)$.

The patient produced many more high velocity movements during this tracking task than control subjects. To quantify this the number of velocity peaks exceeding 30 degrees/sec. was measured for each $20 \mathrm{sec}$. tracking trial. For the patient the mean number of high velocity peaks was $23.1 \pm 2.5$; for control subjects the mean was $8.5 \pm 1$.7. Since the patient lagged further behind the target much of the time during the tracking task, some of these high velocity movements may have represented appropriate attempts to catch up, but at least $50 \%$ were inappropriate, resulting in large target overshoots. 
To obtain an index of the amount by which the subjects' cursor lagged behind the target, the cursor position trace was systematically shifted with respect to target position to determine the point at which the difference between target and cursor reached a minimum value. To minimize tracking error in this manner for the patient required a shift in time of more than 200 msec (mean $224 \pm 30 \mathrm{msec}$ ) indicating that the patient was lagging behind the target by an average of 224 msec. For control subjects the corresponding time shift required to minimize tracking error was $44 \pm 18 \mathrm{msec}$.

The addition of a viscous load to the manipulandum produced a significant improvement in tracking performance for the

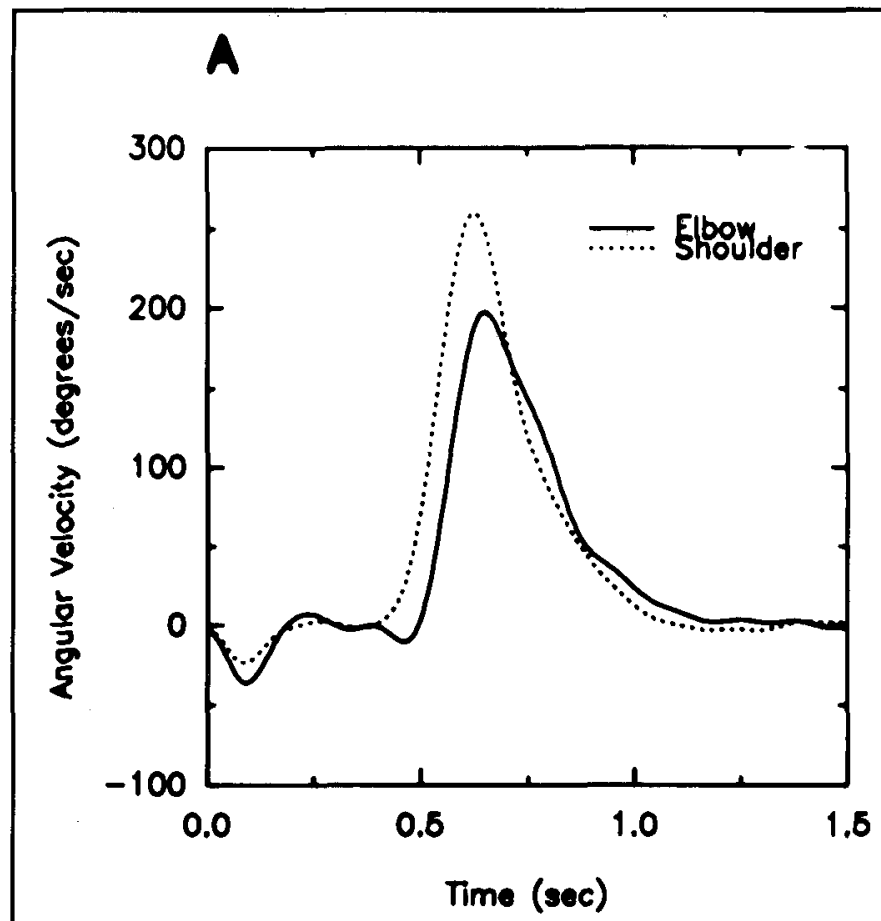

$\mathbf{B}$
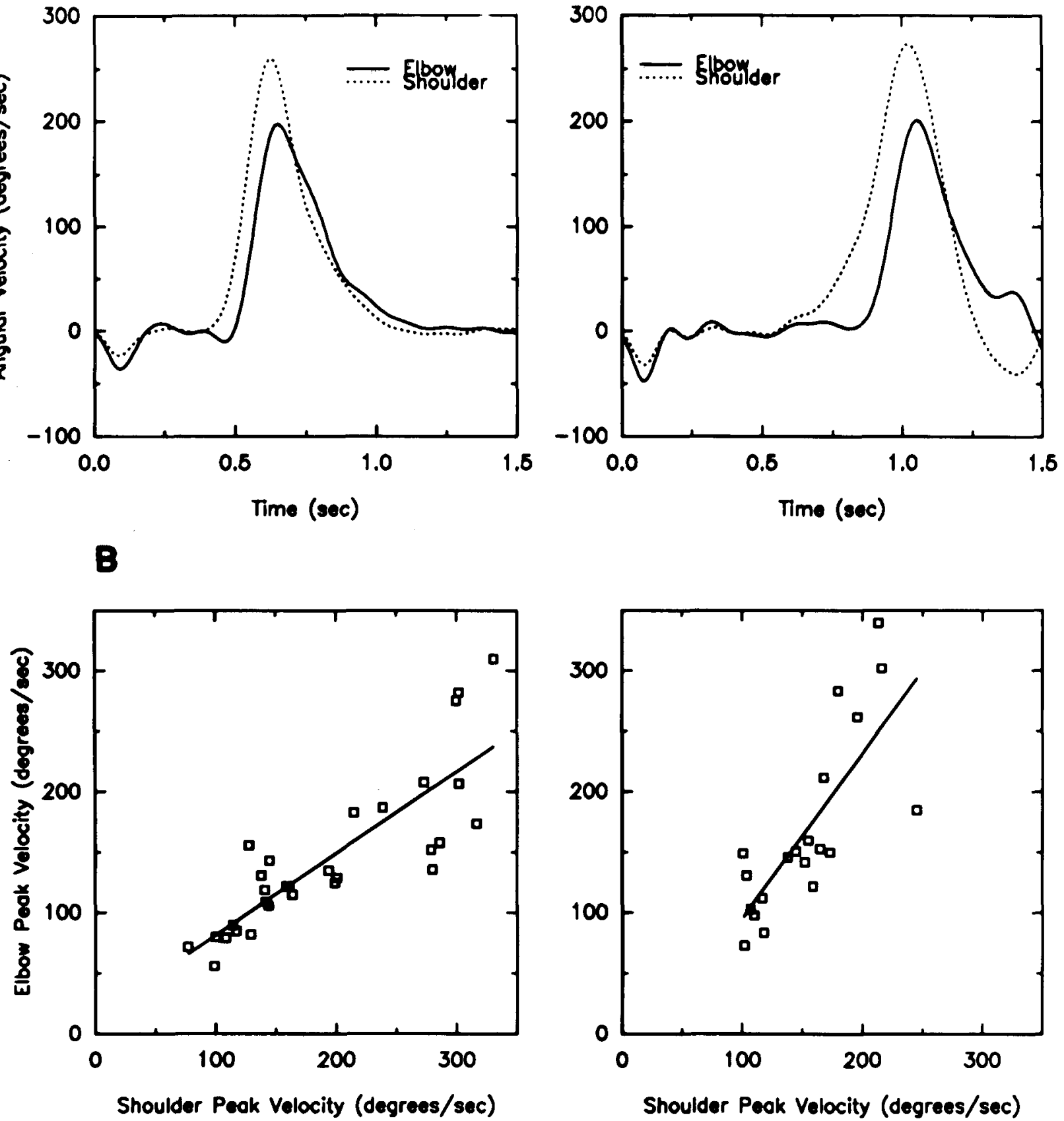

Figure 5-(A) Shoulder and elbow angular velocity profiles during a fast reaching movement for a normal subject (left panel) and for the patient (right panel). Onset of rotation at the elbow occurred later relative to onset of rotation at the shoulder in the patient. (B) In movements with a faster shoulder peak angular velocity, the elbow peak angular velocity was increased relative to slower movements both in normal subjects (left panel) and in the patient (right panel). Correlation coefficients: normal subjects $R=0.84$ ( $n=30$ trials), patient $R=0.77(n=20$ trials $)$. 
patient. Although the positional error score remained much larger than in control subjects, it was reduced from a mean of $152,275 \pm 17,830$ to $134,011 \pm 11,605$ ( $p<0.05$ ) with the viscous load.

The viscous load also resulted in fewer reversals of movement direction during each 20 second tracking trial (29 reversals for loaded trials versus 41 reversals for unloaded trials, $\mathrm{p}<0.005$ ). In addition, the number of high velocity movements was reduced by addition of viscous load. Although this reduction did not reach statistical significance for movements exceeding 30 degrees/sec $(p>0.05)$ it was significant for movements exceeding 50 degrees/sec. In unloaded trials the patient produced an average of $15 \pm 2.0$ movements with peak velocities exceeding 50 degrees/sec. During trials with a viscous load, the corresponding figure was $8.6 \pm 1.8(\mathrm{p}<0.005)$. Adding viscosity had little effect on the amount by which the patient lagged behind the target during tracking. A time shift of $218 \mathrm{msec}$ was still required to reduce tracking error to a minimum value.

In most control subjects, the viscous load did not improve the positional error score or change the number of direction reversals but the number of high velocity movements was reduced.

\section{Discussion}

This patient provided an opportunity to study the control of arm movements in a situation where the modulating effects of Purkinje cells on information transmission through the deep cerebellar nuclei was presumably lost. Abnormalities in control and coordination of movements were apparent for both single joint tracking movements and complex multi-joint movements where the subject was required to reach to a visual target. Although the exact etiology of the cerebellar cortical degeneration in this case remains unknown, the subacute onset and the presence of oligoclonal bands and mononuclear pleocytosis in the cerebrospinal fluid support an autoimmune pathogenesis. The preferential destruction of Purkinje cells and other neuropathologic features were similar to those seen in paraneoplastic cerebellar degeneration in which antibodies directed against Purkinje cell antigens are well documented.3,4 This case cannot be designated "paraneoplastic", however, because of the absence

Figure 6-(A) A shoulder angular velocity profile from a normal subject is shown, with the intervals " $T 1$ " and " $T 2$ " indicated. The onset of $T 1$ is the time at which $50 \%$ peak velocity is reached during acceleration. The end of $T 2$ is the point at which $50 \%$ peak velocity is reached during deceleration. $(B)$ The relationship between interval $T 1$ and shoulder peak velocity is shown for 16 movement trials in the three normal subjects (middle panel) and for 11 trials in the patient (lower panel). For the normal subjects, $R=0.88$. For the patient, $R=0.17$

Figure 7 (opposite page) - Results from a single 20 sec tracking movement trial without load are shown for a control subject (upper panel) and for the patient (middle panel). The lower panel shows, in a similar manner, data from a single trial with a viscous load from the patient. In each panel, changes in position over time are shown for the target (dashed line) and for the wrist (solid line). Similarly, changes in velocity are shown for the target (dashed line) and for the wrist joint (solid line). The solid horizontal lines indicate the neutral wrist position for the position traces and zero velocity for the velocity traces.

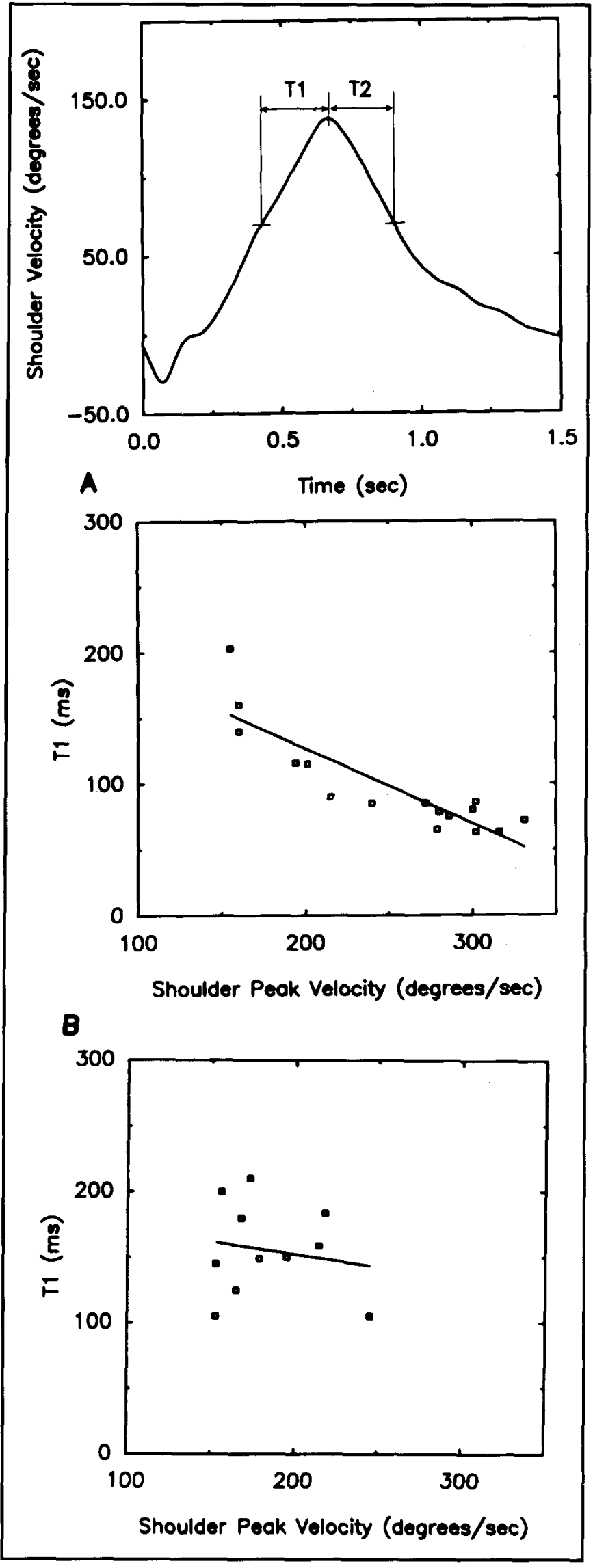

Figure 6 


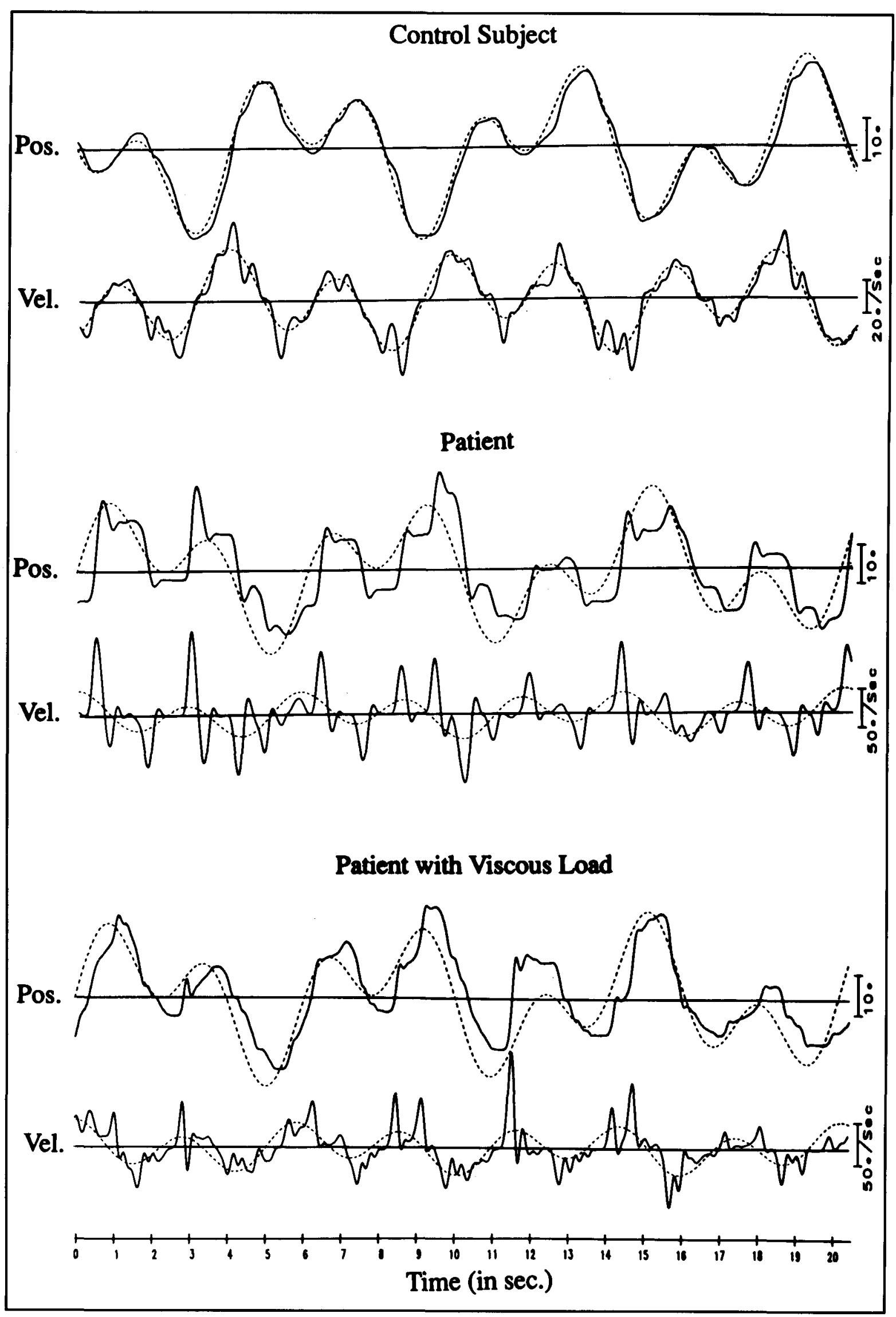

Figure 7 
of evidence of a carcinoma either clinically or at autopsy 8 years after the onset of the neurologic disorder.

\section{Eye-Hand Tracking}

One of the features which contributed to the large tracking error was a tendency for the patient to lag much further behind the target than control subjects. This could be partially accounted for by prolonged visual reaction times which have been reported in patients with cerebellar lesions ${ }^{5}$ and in monkeys following cooling of the deep cerebellar nuclei. ${ }^{6}$ However, a more likely explanation may be that the patient was unable to adopt a predictive strategy to perform this particular type of tracking task. Although the target movement was not easily predictable, the fact that normal subjects lagged behind the target by an average of only $44 \mathrm{msec}$ - a value much less than normal visual reaction time - suggested that they were able to use predictive mechanisms. By contrast the patient tracked the target with a mean delay of more than $200 \mathrm{msec}$.

Inability to control movement velocity was another obvious feature in the tracking records from the patient. There were many inappropriately high velocity movements which caused the patient to overshoot the target. We have observed similar abnormalities in movement velocity during a tracking task in patients with unilateral cerebellar lesions, ${ }^{2}$ and Miall et $\mathrm{al}^{7}$ have described a similar phenomenon in a monkey with temporary cerebellar dysfunction produced by infusion of local anesthetic into the cerebellar cortex. Miall et al suggested that the intact cerebellum may be responsible for "tuning" the raw or primitive movements generated by the motor system and limiting peak velocity.

The sudden decelerations to zero velocity and the often inappropriate brief direction reversals which were a feature of the patient's tracking performance could result from inappropriate stretch reflexes in antagonist muscles during rapid voluntary movements. We cannot confirm this since EMG activity was not recorded as part of this experiment, but there is considerable evidence in the literature to indicate that the cerebellum plays an important role in modulating stretch reflexes according to the requirements of the intended movement. $8,9,10$ Hore and Flament ${ }^{11}$ have proposed that the cerebellum may regulate the manner in which motor cortical neurons respond to stretch of antagonist muscles during voluntary movements. Following cooling of the cerebellar nuclei in monkeys, motor cortical neurons which were previously associated with activation of triceps began to show excessive responses to elbow flexion movements.

The addition of a viscous load improved tracking performance by the patient, partially suppressed the inappropriate high velocity movements, and reduced the number of reversals in movement direction. In some respects the tracking movements made by the patient during unloaded trials suggest a lack of normal damping mechanisms. This could be related to hypotonia which is a common feature of cerebellar dysfunction and which was present in our patient. Added viscosity might partially substitute for the damping normally provided by muscle tone.

In this regard it is interesting to consider experiments on the mutant Lurcher mouse, an animal model for cerebellar degeneration. By 65 days of age these animals have lost virtually all their Purkinje cells, most of the granule cells, and $75 \%$ of the inferior olive cells, but the deep cerebellar nuclei remain intact. Movement control in these mice is considerably better during swimming than during walking ${ }^{12}$ possibly in part because of the increased viscous resistance provided by the water during swimming.

\section{Reaching Movements}

When our normal subjects reached to the visual target, movements at the shoulder and elbow were tightly coupled so that the finger and hand followed approximate straight line paths. These findings are consistent with previous reports. ${ }^{13}$ Loss of this coupling of movements at different joints may be the main factor responsible for "decomposition of movement" in patients with cerebellar damage as described initially by Gordon Holmes. I In our patient elbow extension was often quite delayed with respect to shoulder flexion. In normal subjects performing this pointing task movements at these two joints occurred almost simultaneously. Inability to produce pointing movements with consistent directions from trial to trial was another prominent feature observed in our patient. Similar problems in controlling movement direction have been described in patients with cerebellar cortical degeneration performing a task in which they were required to throw a ball at a target. ${ }^{14}$ These findings indicate a role for the cerebellum in the generation of correct movement directions during reaching to a visual target, and are consistent with experiments in monkeys demonstrating that both the cerebellar cortex and the cerebellar nuclei contain directionally tuned neurons. ${ }^{15}$

Abnormalities of movement velocity which were a prominent feature of single joint movements were also quite apparent in the multi-joint reaching task. Recordings from the patient showed a number of abnormal features in the temporal profiles for angular velocities at the shoulder and elbow. In experiments involving cooling of cerebellar nuclei in monkeys, Flament and Hore ${ }^{16}$ observed that the velocity profile for rapid elbow flexion movements became asymmetric with longer acceleration phases and shorter deceleration phases. In human subjects with a variety of cerebellar disorders, Brown et al ${ }^{17}$ found abnormalities of velocity profiles for single joint target - directed movements.

For the reaching task the location of the target in space is likely encoded by neurons in area 7 of the posterior parietal lobe. Recordings from monkeys have shown that cells in this area encode the location of visual stimuli in head centered space, 18 and removal of areas 5 and 7 results in marked errors during a reaching task to a visual target. ${ }^{19}$ Area 7 , which has many cells with activity related to arm movements, ${ }^{20}$ projects heavily to the pontine nuclei which in turn send projections to the cerebellar cortex. ${ }^{21}$ The major output projections from the cerebellum originate from the deep nuclei and many of these go to motor cortex. Selective damage to the inhibitory Purkinje cells could create a situation in which the deep nuclei would exert their influence on target neurons in cerebral cortex and other areas without the modulating influences of the cerebellar cortex.

How might this result in the abnormalities which we observed in our patient? Visual information from the posterior parietal area, some of which may relate to target location, may be transmitted to the cerebellum via the pontine nuclei. Recordings from monkeys have indicated that many cells in the dentate nucleus are responsive to visual stimuli.22,23 It is possible that the cerebellum normally plays a major role in integrating visual information concerning target location with the intended limb movement to the target. With loss of the modulating effect of Purkinje cells on deep cerebellar nuclei, the signals transmitted from the cerebellar nuclei to motor cortex could produce inappropriate motor output causing errors in direction, velocity, and other properties of movement. 
Our findings are consistent with the view that neuronal pathways which run through the cerebellar cortex contribute to a number of different aspects of motor coordination. These likely include sensorimotor integration mechanisms which adjust motor output in response to relevant visual information.

\section{ACKNOWLEDGEMENTS}

This research was funded by the Medical Research Council of Canada and the Alberta Heritage Foundation for Medical Research.

\section{REFERENCES}

1. Holmes G. The cerebellum of man. Brain 1939; 62: 1-30.

2. Morrice BL, Becker WJ, Hoffer JA, et al. Manual tracking performance in patients with cerebellar incoordination: Effects of mechanical loading. Can J Neurol Sci 1990; 17: 275-285.

3. Anderson NE, Rosenblum MK, Posner JB. Paraneoplastic cerebellar degeneration: Clinical-immunological correlations. Ann Neurol 1988; 24: 559-567.

4. Furneaux HM, Rosenblum MK, Dalmau J, et al. Selective expression of Purkinje-cell antigens in tumor tissue from patients with paraneoplastic cerebellar degeneration. N Engl J Med 1990; 322: 1844-1851.

5. Beppu H, Suda M, Tanaka R. Analysis of cerebellar motor disorders by visually guided elbow tracking movement. Brain 1984; 107: 787-809.

6. Meyer-Lohmann J, Hore J, Brooks VB. Cerebellar participation in generation of prompt arm movements. J Neurophysiol 1977; 40: 1038-1050.

7. Miall RC, Weir DJ, Stein JF. Visuo-motor tracking during reversible inactivation of the cerebellum. Exp Brain Res 1987; 65: 455-464.

8. Thach WT, Perry JG, Schieber MH. Cerebellar output: Body maps and muscle spindles. Exp Brain Res 1982, Suppl. 6; SpringerVerlag, Berlin-Heidelberg, FRG.

9. MacKay WA, Murphy JT. Cerebellar modulation of reflex gain. Prog Neurobiol 1979; 13: 361-417.
10. Hore $\mathrm{J}$, Villis $\mathrm{T}$. Loss of set in muscle responses to limb perturbations during cerebellar dysfunction. J Neurophysiol 1984; 51 : 1137-1147.

11. Hore J, Flament D. Changes in motor cortex neural discharge associated with the development of cerebellar limb ataxia. J Neurophysiol 1988; 60: 1285-1302.

12. Fortier PA, Smith AM, Rossignol S. Locomotor deficits in the mutant mouse, Lurcher. Exp Brain Res 1987; 66: 271-286.

13. Soechting JF. Laquaniti $F$. Invariant characteristics of a pointing movement in man. $\mathrm{J}$ Neurosci $1981 ; 1: 710-720$

14. Becker WJ, Kunesch E, Freund H-J. Coordination of a multi-joint movement in normal humans and in patients with cerebellar dysfunction. Can J Neurol Sci 1990; 17: 264-274.

15. Fortier PA, Kalaska JF, Smith AM. Cerebellar neuronal activity related to whole arm reaching movements in the monkey. J Neurophysiol 1989; 62: 198-211.

16. Flament D, Hore J. Movement and electromyographic disorders associated with cerebellar dysmetria. J Neurophysiol 1986; 55: 1221-1233.

17. Brown SH, Hefter $\mathbf{H}$, Mertens M, et al. Disturbances in human arm movement trajectory due to mild cerebellar dysfunction. J Neurol Neurosurg Psychiatry 1990; 53: 306-313.

18. Anderson RA, Essick GK, Siegel RM. Encoding of spatial location by posterior parietal neurons. Science $1985 ; 230: 456-458$.

19. Lamotte RH, Acuna C. Defects in accuracy of reaching after removal of posterior parietal cortex in monkeys. Brain Res 1978; 139: 309-326.

20. Kalaska JF. The representation of arm movements in post-central and parietal cortex. Can J Physiol Pharmacol 1988; 66: 455-463.

21. Glickstein M, May III JG, Mercier BE. Corticopontine projection in the Macaque: The distribution of labelled cortical cells after large injections of horse radish peroxidase in the pontine nuclei. J Comp Neurol 1985; 235: 343-359.

22. Chapman EC, Spidalieri G, Lamarre Y. Activity of dentate neurons during arm movements triggered by visual, auditory and somesthetic stimuli in the monkey. J Neurophysiol 1986; 55: 203-225.

23. MacKay WA. Unit activity in the cerebellar nuclei related to arm reaching movements. Brain Res 1988; 442; 240-254. 